\title{
Gamma-ray Observation of SNR G106.3+2.7 with the Tibet Air Shower Array
}

\section{Ohnishi* on behalf of the Tibet AS $\gamma$ Collaboration}

(a complete list of authors can be found at the end of the proceedings)

We report the observation of gamma-ray emission from the supernova remnant G106.3+2.7 in the $100 \mathrm{TeV}$ energy region with the Tibet air shower array combined with the muon detector array. The gamma-ray emission is detected at a significance of $6.1 \sigma$ above $10 \mathrm{TeV}$. The centroid of the gamma-ray emission, situated $0.44^{\circ}$ away from the pulsar PSR J2229+6114 at the north-east corner of G106.3+2.7, is found to be coincident with the location of a molecular cloud. This morphological feature favors a hadronic origin via the $\pi^{0}$ decay caused by accelerated relativistic protons regarding the gamma-ray emission mechanism of G106.3+2.7, over a leptonic one via the inverse-Compton scattering by relativistic electrons. The differential gamma-ray energy spectrum can be expressed by a single power law from $6 \mathrm{TeV}$ to $115 \mathrm{TeV}$ as $d N / d E=N_{0}(E / 40 \mathrm{TeV})^{-\Gamma}$ with a flux of $N_{0}=(9.5 \pm 1.6) \times 10^{-16}\left[\mathrm{~cm}^{-2} \mathrm{~s}^{-1} \mathrm{TeV}^{-1}\right]$ and an index of $\Gamma=2.95 \pm 0.17$. In the hadronic scenario, we estimate the cut-off energy of parent cosmic-ray particles (protons) to be around $0.5 \mathrm{PeV}$.

$37^{\text {th }}$ International Cosmic Ray Conference (ICRC 2021)

July 12th-23rd, 2021

Online - Berlin, Germany

\footnotetext{
*Presenter
} 


\section{Introduction}

The supernova remnant (SNR) G106.3+2.7 is an extended comet-shaped source discovered in the northern Galactic Plane survey by the Dominion Radio Astrophysical Observatory [1]. Located at the northeast edge of G106.3+2.7 is the so-called "Boomerang" pulsar wind nebula (PWN) G106.65+2.96, which is powered by an energetic $\left(\dot{E}=2.2 \times 10^{37} \mathrm{erg} \mathrm{s}^{-1}\right)$ pulsar PSR J2229+6114 of age $10.5 \mathrm{kyr}[2,3]$. The radio and X-ray pulsations of PSR J2229+6114 were observed with a period of $51.6 \mathrm{~ms}$ [4]. The gamma-ray counterpart of PSR J2229+6114 was detected and named 0FGL J2229.0+6114 by the Fermi Large Area Telescope [5], and its $\gamma$-ray pulsations above $100 \mathrm{MeV}$ were detected by Fermi [6] and AGILE [7]. Upper limits were given on the flux of PWN G106.65+2.96 between $100 \mathrm{GeV}$ and $1 \mathrm{TeV}$ by the MAGIC experiment [8]. As discussed in [9], PSR J2229+6114 and SNR G106.3+2.7 could be the result of the same supernova explosion, and the radial velocity measurements of molecular material and atomic hydrogen suggested a distance of $\sim 0.8 \mathrm{kpc}$ to the whole system.

The Milagro experiment reported an elongated gamma-ray source MGRO J2228+61 coincident with PSR J2229+6114 at $35 \mathrm{TeV}$ [10, 11]. Meanwhile, the VERITAS experiment detected gammaray emissions above $1 \mathrm{TeV}$ from the supernova remnant (SNR) G106.3+2.7 with a flux of $\sim 5 \%$ Crab and named the source VER J2227+608 [12]. Recently the HAWC experiment observed G106.3+2.7 and reported a best-fit spectrum with an error band above $40 \mathrm{TeV}$ [13]. The centroid of VER J2227+608, 0.4 ${ }^{\circ}$ away from PSR J2229+6114 in the southwest direction, is consistent with that of MGRO J2228+61 and the HAWC centroid within statistical and systematic uncertainties. Fermi also observed this source at $\mathrm{GeV}$ energies and found that the source location is coincident with that of the molecular cloud [14].

\section{Experiment and Data Analysis}

The Tibet air-shower (AS) array has been observing cosmic rays and gamma rays above $\mathrm{TeV}$ energies since 1990 at Yangbajing $\left(90.522^{\circ} \mathrm{E}, 30.102^{\circ} \mathrm{N} ; 4300 \mathrm{~m}\right.$ above sea level) in Tibet, China [15]. In this work, we use data obtained by the Tibet air shower array combined with the muon detector array during 719 live days from 2014 February to 2017 May to observe high-energy gamma-ray emissions from the region around G106.3+2.7. Our data analysis method and data selection criteria as well as the array configuration are the same as described in our previous papers [16].

\section{Results and Discussions}

Figure 1 shows the detection significance map around G106.3+2.7 above $10 \mathrm{TeV}$, smoothed by the search window size. The events can be fitted with a symmetrical 2D Gaussian function, and the centroid of gamma-ray emissions (a red filled star with a red position error circle) is at (R.A., Dec.) $=\left(336.82^{\circ} \pm 0.16_{\text {stat }}^{\circ}, 60.85^{\circ} \pm 0.10_{\text {stat }}^{\circ}\right)$, coincident with a nearby molecular cloud location revealed by ${ }^{12} \mathrm{CO}(J=1-0)$ emissions (green contours) [20] overlying the black radio contours [18, 19] of the SNR and away from PSR J2229+6114 by $0.44^{\circ}$ in the southwest direction. Our source location is also consistent with those of VERITAS [12] and HAWC [13]. Given the distance of $800 \mathrm{pc}$ 
from the Earth to both PSR J2229+6114 and SNR G106.3+2.7, the distance from the pulsar to the source location obtained by this work is estimated at $6 \mathrm{pc}$. Our source location deviates from the pulsar location at a confidence level of $3.1 \sigma$, based on the error of $0.14^{\circ}$ including both statistical and systematic errors. The location of the HAWC centroid is consistent with both those of the Boomerang pulsar and the molecular cloud location, and the centroids of VERITAS and Fermi are coincident with the location of molecular cloud as well as our source location.

Figure 2 shows the distribution of the number of observed events above $10 \mathrm{TeV}$ as a function of the opening angle between the measured arrival direction and our source location. Fitting the data with a Gaussian function $A \exp \left(-\phi^{2} /\left(2\left(\sigma_{\mathrm{PSF}}^{2}+\sigma_{\mathrm{EXT}}^{2}\right)\right)\right)+N_{\mathrm{BG}}$ where $\sigma_{\mathrm{PSF}}=0.35^{\circ}$ is our point spread function above $10 \mathrm{TeV}$ and $N_{\mathrm{BG}}=148$ is the number of background events, we estimate the $1 \sigma$ extent of the source to be $\sigma_{\mathrm{EXT}}=0.24^{\circ} \pm 0.10_{\text {stat }}^{\circ}$, consistent with that estimated by VERITAS of $0.27^{\circ}\left(0.18^{\circ}\right)$ along the major (minor) axis.

Figure 3 shows the differential gamma-ray energy spectrum measured by this work (red filled squares and two red downward arrows for two upper limits). The detection significance above $10 \mathrm{TeV}$ is $6.1 \sigma$. Our gamma-ray energy spectrum can be fitted by a single power law from 6 to $115 \mathrm{TeV}$ as $d N / d E=N_{0}(E / 40 \mathrm{TeV})^{-\Gamma}$ with $N_{0}=\left(9.5 \pm 1.6_{\text {stat }}\right) \times 10^{-16}\left[\mathrm{~cm}^{-2} \mathrm{~s}^{-1} \mathrm{TeV}^{-1}\right]$ and $\Gamma=2.95 \pm 0.17_{\text {stat }}\left(\chi^{2} / \mathrm{ndf}=2.5 / 5\right)$. The systematic error of $N_{0}$ is estimated to be $+40 \% /-31 \%$, resulting from the $12 \%$ uncertainty in the absolute energy scale determination. Our spectrum is consistent with the HAWC spectrum. The flux data points of VERITAS (blue filled circles) are raised by a factor of 1.62 to account for the spill-over of gamma-ray signals outside their window size. Our three flux data points below $20 \mathrm{TeV}$ overlapping the energy range covered by the VERITAS flux points are statistically consistent with 1.62 times VERITAS's original best-fit power-law function reported in their paper [12] at the $1.5 \sigma$ level.

As to the physical mechanism of the gamma-ray emission we fit the multi-wavelength gammaray energy spectrum using the naima package [21], which allows us to estimate the parent particle spectrum so as to best reproduce the observed gamma-ray energy spectrum. For the energy distribution of the parent particles, we assume an exponential cut-off power-law form of $d N / d E \propto$ $E^{-\alpha} \exp \left(-E / E_{\text {cut }}\right)$. The best-fit parameters are listed in Table 1 . In the hadronic model, we get $E_{\text {cut }} \sim 0.5 \mathrm{PeV}$ and $\alpha \sim 1.8$. The value of $\alpha$ falls between that predicted in the standard diffusive shock acceleration $(\alpha=2)$ and the asymptotic limit of the very efficient proton acceleration $(\alpha=1.5)$ [22, 23]. The total energy of protons with energies $>1 \mathrm{GeV}(>0.5 \mathrm{PeV})$ is estimated to be $\sim 5.0 \times 10^{47} \mathrm{erg}\left(3.0 \times 10^{46} \mathrm{erg}\right)$ for a target gas density of $10 \mathrm{~cm}^{-3}$. One might argue that, considering the estimated SNR age of $10 \mathrm{kyr}, \mathrm{PeV}$ protons escape the SNR much earlier than the present time in the standard theory of cosmic-ray acceleration. Given that $E_{\text {cut }} \sim 0.5 \mathrm{PeV}$ and that the maximum energy of protons remaining inside an SNR is proportional to $\tau^{-0.5}$ where $\tau$ is the SNR age[24], protons should be accelerated up to $\sim 1.6 \mathrm{PeV}$ at $\tau=1 \mathrm{kyr}$ in the case of G106.3+2.7. This suggests that the acceleration of protons at G106.3+2.7 should be efficient enough[24] to push their maximum energy up to $\sim 1.6 \mathrm{PeV}$ during the SNR free expansion phase. In addition, G106.3+2.7 has a dense molecular cloud nearby indispensable for accelerated protons to produce $\mathrm{TeV}$ gamma rays via $\pi^{0}$ production. With $\alpha \sim 1.8$, the proton energy spectrum does not appear softened, implying that protons may not be able to escape the SNR easily due to the suppression of the diffusion coefficient. Future observations of G106.3+2.7 could provide useful information for these theoretical studies on its mechanisms of particle acceleration and confinement. 
In the leptonic model, we get $E_{\text {cut }} \sim 190 \mathrm{TeV}, \alpha \sim 2.3$ and the SNR magnetic field strength of $\sim 9 \mu \mathrm{G}$. The total energy of relativistic electrons with energies $>10 \mathrm{MeV}$ is estimated to be $\sim 1.4 \times 10^{47} \mathrm{erg}$. Considering the synchrotron cooling, we estimate that electrons need to be accelerated freshly within $1 \mathrm{kyr}$ if they originate from the SNR, and that electrons provided by the Boomerang PWN are not likely to produce the observed gamma-ray emission in view of the energy budget and the gamma-ray morphology.

Generally, the energy spectrum of hadronically-induced gamma rays rises steeply below $\sim 200 \mathrm{MeV}$ and approximately follows the energy spectrum of parent particles above a few $\mathrm{GeV}$, resulting in a characteristic " $\pi^{0}$-decay bump" in the gamma-ray spectrum. Hopefully, future multiwavelength observations would establish the hadronic origin of gamma-ray emissions from SNR G106.3+2.7.

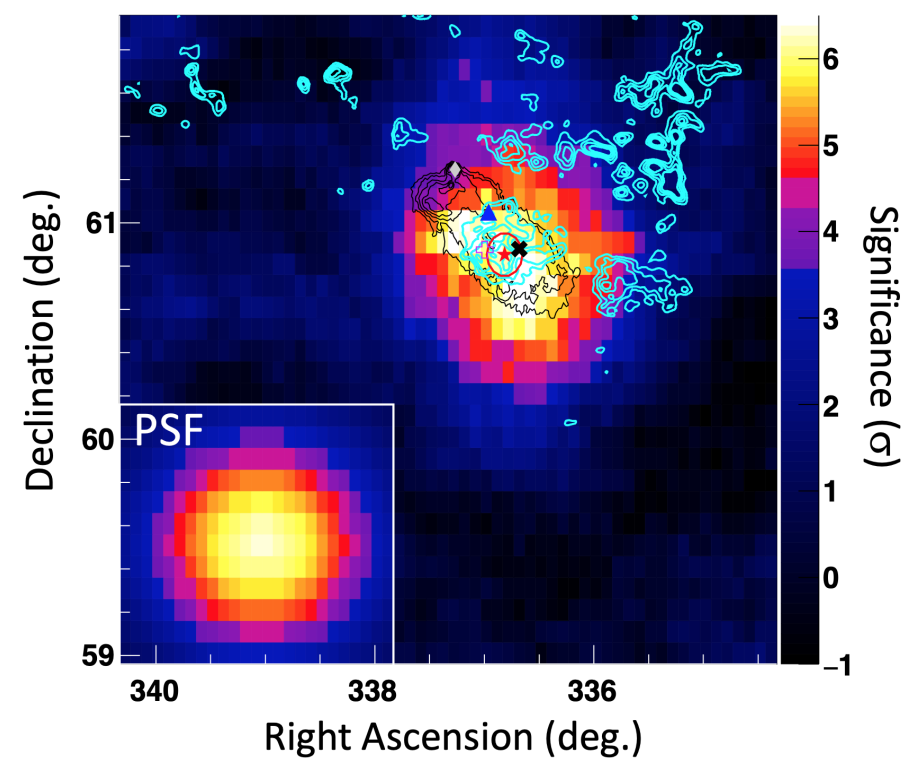

Figure 1: Detection significance map around SNR G106.3+2.7 observed by Tibet AS+MD above $10 \mathrm{TeV}$ [17]. The red filled star with a $1 \sigma$ statistical position error circle is our source location, while the magenta open cross, the black X mark and the blue filled triangle are the centroids determined by VERITAS [12], Fermi [14] and HAWC [13]. The black contours represent $1420 \mathrm{MHz}$ radio emissions from the Dominion Radio Astrophysical Observatory Synthesis Telescope [18, 19], and the cyan contours represent ${ }^{12} \mathrm{CO}$ emissions from the Five College Radio Astronomy Observatory survey [20]. The gray filled diamond at the northeast corner of the black contours indicates the pulsar PSR J2229+6114. The inset figure shows our point spread function (PSF). 


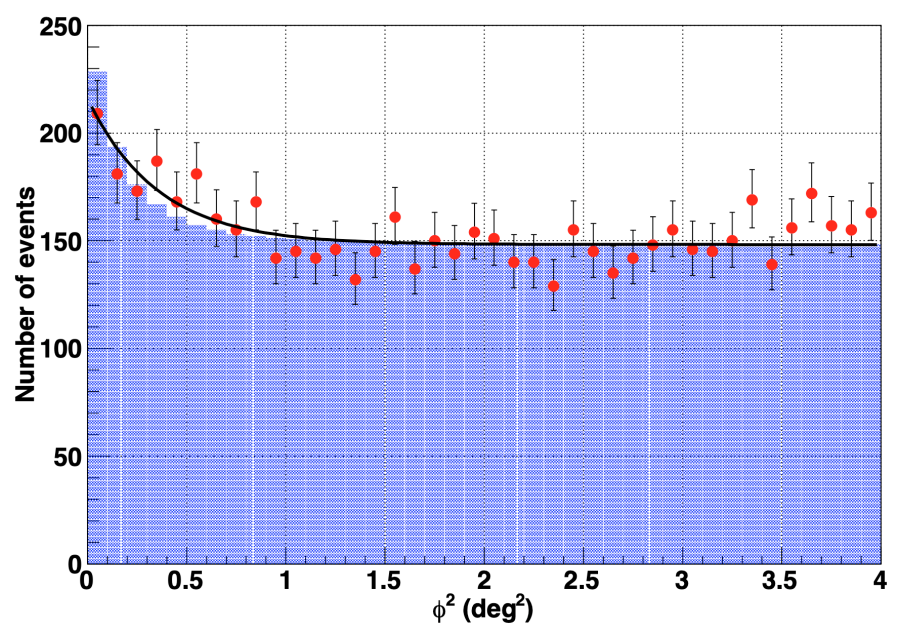

Figure 2: Projected angular distribution of events observed above $10 \mathrm{TeV}$ [17]. The horizontal axis $\phi^{2}$ is the square of the opening angle between the estimated event arrival direction and our source location. The red filled circles are the experimental data with the best-fit black solid curve. The purple histogram is the expected event distribution by MC simulations assuming a point-like gamma-ray source.

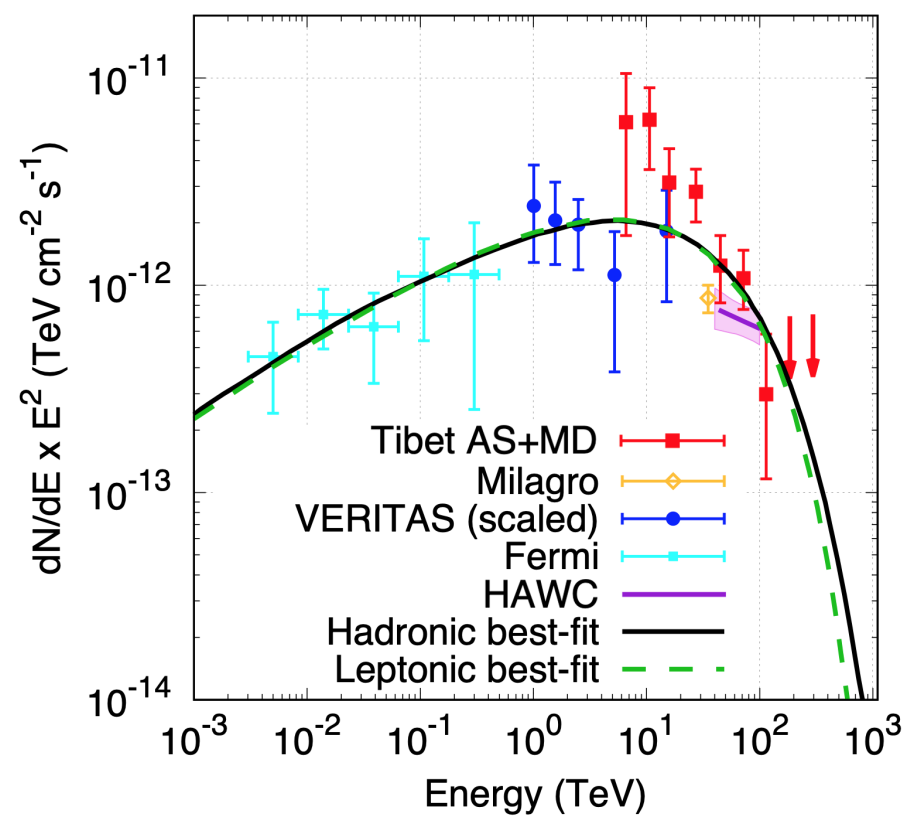

Figure 3: Differential gamma-ray energy spectrum of SNR G106.3+2.7 [17]. Red filled squares (Tibet AS+MD) represent data measured by this work with two $99 \%$ C.L. upper limits (downward red arrows), VERITAS [12] (deep-blue filled circles), Fermi [14] (sky-blue crosses), Milagro [11] (an orange open diamond) and HAWC [13] (a purple solid line with a shaded light purple area indicating the $1 \sigma$ statistical error band). VERITAS's data points are raised by a factor of 1.62 from the original values (see text). The black solid (green dashed) line is the best-fit curve of the hadronic (leptonic) model for the combined data points of Tibet AS+MD, VERITAS and Fermi. 
Table 1: Best-fit parameters of the energy distribution of parent particles [17]. Electrons (protons) are assumed for the parent particles of the leptonic (hadronic) model.

\begin{tabular}{cccccc}
\hline & $\alpha$ & $E_{\text {cut }}(\mathrm{TeV})$ & $W_{e / p}^{\ddagger}\left(10^{47} \mathrm{erg}\right)$ & $B(\mu \mathrm{G})$ & $\chi^{2} / \mathrm{ndf}$ \\
\hline leptonic & $2.30_{-0.07}^{+0.08}$ & $190_{-66}^{+127}$ & $1.4_{-0.7}^{+1.8}$ & $8.6_{-2.5}^{+3.4}$ & $12.8 / 15$ \\
hadronic & $1.79_{-0.09}^{+0.08}$ & $499_{-180}^{+382}$ & $5.0_{-0.6}^{+0.7}$ & - & $13.0 / 14$
\end{tabular}

${ }^{\ddagger} W_{e / p}$ is the total energy above $10 \mathrm{MeV}$ and $1 \mathrm{GeV}$ for electrons and protons, respectively, where $W_{p}$ is for a target gas density of $10 \mathrm{~cm}^{-3}$.

\section{Acknowledgements}

The collaborative experiment of the Tibet Air Shower Arrays has been conducted under the auspices of the Ministry of Science and Technology of China and the Ministry of Foreign Affairs of Japan. This work was supported in part by a Grant-in-Aid for Scientific Research on Priority Areas from the Ministry of Education, Culture, Sports, Science and Technology, and by Grants-in-Aid for Science Research from the Japan Society for the Promotion of Science in Japan. This work is supported by the National Key R\&D Program of China (No. 2016YFE0125500), the Grants from the National Natural Science Foundation of China (Nos. 11533007, 11673041, 11873065, 11773019, 11773014, 11633007, 11803011, and 11851305), and the Key Laboratory of Particle Astrophysics, Institute of High Energy Physics, CAS. This work is also supported by the joint research program of the Institute for Cosmic Ray Research (ICRR), the University of Tokyo.

\section{References}

[1] G. Joncas \& L. A. Higgs, Astron. Astrophys. Suppl. Ser. 82, 113 (1990).

[2] R. C. Hartman et al., Astrophys. J. Suppl. Ser. 123, 79 (1999).

[3] Manchester, R. N., Hobbs, G. B., Teoh, A., \& Hobbs, M., Astron. J. 129, 1993 (2005).

[4] J. P. Halpern et al., Astrophys. J. 552, L125 (2001).

[5] A. A. Abdo et al., Astrophys. J. Suppl. Ser. 183, 46 (2009).

[6] A. A. Abdo et al., Astrophys. J. 706, 1331 (2009).

[7] Pellizzoni, A., et al., Astrophys. J. 695, L115 (2009).

[8] H. Anderhub et al., Astrophys. J. 710, 828 (2010).

[9] R. Kothes, B. Uyaniker \& S. Pineault, Astrophys. J. 560, 236 (2001).

[10] A. A. Abdo et al., Astrophys. J. 664, L91 (2007).

[11] A. A. Abdo et al., Astrophys. J. 700, L127 (2009).

[12] V. A. Acciari et al., Astrophys. J. 703, L6 (2009).

[13] A. Albert et al., Astrophys. J. 896, L29 (2020).

[14] Y. Xin, H. Zeng, S. Liu, Y. Fan \& D. Wei, Astrophys. J. 885, 162 (2019).

[15] M. Amenomori et al., Phys. Rev. Let. 69, 2468 (1992).

[16] M. Amenomori et al., Phys. Rev. Let. 123, 051101 (2019).

[17] M. Amenomori et al., Nat. Astron. Let. 5, 460 (2021).

[18] A. R. Taylor et al., Astron. J. 125, 3145 (2003).

[19] T. L. Landecker et al., Astron. Astrophys. Suppl. Ser. 145, 509 (2000).

[20] M. H. Heyer et al., Astrophys. J. Suppl. Ser. 115, 241 (1998).

[21] V. Zabalza, https://arxiv.org/abs/1509.03319 (2015). 
[22] M. A. Malkov, Astrophys. J. 511, L53 (1999).

[23] E. G. Berezhko \& D. C. Ellison, Astrophys. J. 526, 385 (1999).

[24] D. Caprioli, P. Blasi \& E. Amato, Mon. Not. R. Astron. Soc. 396, 2065 (2009). 


\section{Full Authors List: The Tibet AS $\gamma$ Collaboration}

M. Amenomori ${ }^{1}$, S. Asano ${ }^{2}$, Y. W. Bao ${ }^{3}$, X. J. Bi ${ }^{4}$, D. Chen ${ }^{5}$, T. L. Chen ${ }^{6}$, W. Y. Chen ${ }^{4}, \mathrm{Xu} \mathrm{Chen}^{4}, \mathrm{Y}_{\mathrm{C}} \mathrm{Chen}^{3}$, $\mathrm{Cirennima}^{6}$, S. W. Cui ${ }^{7}$,

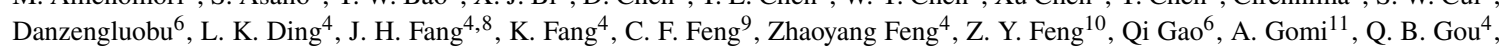
Y. Q. Guo ${ }^{4}$, Y. Y. Guo ${ }^{4}$, H. H. He ${ }^{4}$, Z. T. He ${ }^{7}$, K. Hibino ${ }^{12}$, N. Hotta ${ }^{13}$, Haibing Hu${ }^{6}$, H. B. Hu ${ }^{4}$, K. Y. Hu $u^{4,8}$, J. Huang ${ }^{4}$, H. Y. Jia ${ }^{10}$, L. Jiang ${ }^{4}$, P. Jiang ${ }^{5}$, H. B. Jin ${ }^{5}$, K. Kasahara ${ }^{14}$, Y. Katayose ${ }^{11}$, C. Kato ${ }^{2}$, S. Kato ${ }^{15}$, T. Kawashima ${ }^{15}$, K. Kawata ${ }^{15}$, M. Kozai ${ }^{16}$, D. Kurashige ${ }^{11}$, Labaciren ${ }^{6}$, G. M. Le ${ }^{17}$, A. F. $\mathrm{Li}^{18,9,4}$, H. J. Li ${ }^{6}$, W. J. Li ${ }^{4,10}$, Y. Li ${ }^{5}$, Y. H. Lin ${ }^{4,8}$, B. $\mathrm{Liu}^{19}$, C. Liu ${ }^{4}$, J. S. $\mathrm{Liu}^{4}$,

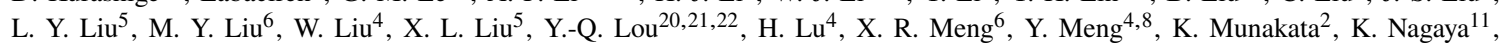
Y. Nakamura ${ }^{15}$, Y. Nakazawa ${ }^{23}$, H. Nanjo ${ }^{1}$, C. C. $\mathrm{Ning}^{6}$, M. Nishizawa ${ }^{24}$, M. Ohnishi ${ }^{15}$, S. Okukawa ${ }^{11}$, S. Ozawa ${ }^{25}$, L. Qian $^{5}$, X. Qian ${ }^{5}$, X. L. Qian ${ }^{26}$, X. B. Qu $^{27}$, T. Saito ${ }^{28}$, Y. Sakakibara ${ }^{11}$, M. Sakata ${ }^{29}$, T. Sako ${ }^{15}$, T. K. Sako ${ }^{15}$, J. Shao ${ }^{4,9}$, M. Shibata ${ }^{11}$, A. Shiomi ${ }^{23}$, H. Sugimoto ${ }^{30}$, W. Takano ${ }^{12}$, M. Takita ${ }^{15}$, Y. H. Tan ${ }^{4}$, N. Tateyama ${ }^{12}$, S. Torii ${ }^{31}$, H. Tsuchiya $^{32}$, S. Udo $^{12}$, H. Wang $^{4}$, Y. P. Wang ${ }^{6}$, Wangdui ${ }^{6}$, H. R. $\mathrm{Wu}^{4}$, Q. $\mathrm{Wu}^{6}$, J. L. $\mathrm{Xu}^{5}$, L. Xue ${ }^{9}$, Z. Yang ${ }^{4}$, Y. Q. Yao ${ }^{5}$, J. Yin ${ }^{5}$, Y. Yokoe ${ }^{15}$, N. P. Yu ${ }^{5}$, A. F. Yuan ${ }^{6}$, L. M. Zhai ${ }^{5}$, C. P. Zhang ${ }^{5}$, H. M. Zhang ${ }^{4}$, J. L. Zhang ${ }^{4}$, X. Zhang ${ }^{3}$, X. Y. Zhang ${ }^{9}$, Y. Zhang ${ }^{4}$, Yi Zhang ${ }^{33}$, Ying Zhang ${ }^{4}$, S. P. Zhao ${ }^{4}$, Zhaxisangzhu $^{6}$ and X. X. Zhou ${ }^{10}$

${ }^{1}$ Department of Physics, Hirosaki University, Hirosaki 036-8561, Japan. ${ }^{2}$ Department of Physics, Shinshu University, Matsumoto 390-8621, Japan. ${ }^{3}$ School of Astronomy and Space Science, Nanjing University, Nanjing 210093, China. ${ }^{4}$ Key Laboratory of Particle Astrophysics, Institute of High Energy Physics, Chinese Academy of Sciences, Beijing 100049, China. ${ }^{5}$ National Astronomical Observatories, Chinese Academy of Sciences, Beijing 100012, China. ${ }^{6}$ Department of Mathematics and Physics, Tibet University, Lhasa 850000, China. ${ }^{7}$ Department of Physics, Hebei Normal University, Shijiazhuang 050016, China. ${ }^{8}$ University of Chinese Academy of Sciences, Beijing 100049, China. ${ }^{9}$ Institute of Frontier and Interdisciplinary Science and Key Laboratory of Particle Physics and Particle Irradiation (MOE), Shandong University, Qingdao 266237, China. ${ }^{10}$ Institute of Modern Physics, SouthWest Jiaotong University, Chengdu 610031, China. ${ }^{11}$ Faculty of Engineering, Yokohama National University, Yokohama 240-8501, Japan. ${ }^{12}$ Faculty of Engineering, Kanagawa University, Yokohama 221-8686, Japan. ${ }^{13}$ Faculty of Education, Utsunomiya University, Utsunomiya 3218505, Japan. ${ }^{14}$ Faculty of Systems Engineering, Shibaura Institute of Technology, Omiya 330-8570, Japan. ${ }^{15}$ Institute for Cosmic Ray Research, University of Tokyo, Kashiwa 277-8582, Japan. ${ }^{16}$ Institute of Space and Astronautical Science, Japan Aerospace Exploration Agency (ISAS/JAXA), Sagamihara 252-5210, Japan. ${ }^{17}$ National Center for Space Weather, China Meteorological Administration, Beijing 100081, China. ${ }^{18}$ School of Information Science and Engineering, Shandong Agriculture University, Taian 271018, China. ${ }^{19}$ Department of Astronomy, School of Physical Sciences, University of Science and Technology of China, Hefei 230026, China. ${ }^{20}$ Department of Physics and Tsinghua Centre for Astrophysics (THCA), Tsinghua University, Beijing 100084, China. ${ }^{21}$ Tsinghua University-National Astronomical Observatories of China (NAOC) Joint Research Center for Astrophysics, Tsinghua University, Beijing 100084, China. ${ }^{22}$ Department of Astronomy, Tsinghua University, Beijing 100084, China. ${ }^{23}$ College of Industrial Technology, Nihon University, Narashino 275-8576, Japan. ${ }^{24}$ National Institute of Informatics, Tokyo 101-8430, Japan. ${ }^{25}$ National Institute of Information and Communications Technology, Tokyo 184-8795, Japan. ${ }^{26}$ Department of Mechanical and Electrical Engineering, Shangdong Management University, Jinan 250357, China. ${ }^{27}$ College of Science, China University of Petroleum, Qingdao 266555, China. ${ }^{28}$ Tokyo Metropolitan College of Industrial Technology, Tokyo 116-8523, Japan. ${ }^{29}$ Department of Physics, Konan University, Kobe 658-8501, Japan. ${ }^{30}$ Shonan Institute of Technology, Fujisawa 251-8511, Japan. ${ }^{31}$ Research Institute for Science and Engineering, Waseda University, Tokyo 162-0044, Japan. ${ }^{32}$ Japan Atomic Energy Agency, Tokai-mura 319-1195, Japan. ${ }^{33}$ Key Laboratory of Dark Matter and Space Astronomy, Purple Mountain Observatory, Chinese Academy of Sciences, Nanjing 210034, China. 